\title{
Rsad2 is necessary for mouse dendritic cell maturation via the IRF7-mediated signaling pathway
}

\author{
Ji-Su Jang', Jun-Ho Lee ${ }^{1,2}$, Nam-Chul Jung², So-Yeon Choi', Soo-Yeoun Park', Ji-Young Yoo', Jie-Young Song $\mathbb{1}^{3}$, \\ Han Geuk Seo ${ }^{4}$, Hyun Soo Lee ${ }^{2}$ and Dae-Seog Lim ${ }^{1}$
}

\begin{abstract}
Dendritic cells (DCs) are the most potent professional antigen presenting cells and inducers of T cell-mediated immunity. However, few specific markers of mature DCs (mDC) have been reported. A previous microarray analysis revealed expression of mDC-specific genes and identified Rsad2 (radical S-adenosyl methionine domain containing 2) as a candidate specific marker for DC maturation. Mouse bone marrow-derived DCs were transfected with Rsad2 siRNA and examined by flow cytometry, ELISA, western, and confocal microscopy. C57BL/6 mice received intravenously B16F10 cells to establish a pulmonary metastasis model. Tumor-bearing mice then received subcutaneously two injections of mDCs or Rsad2 knockdown DCs. The cytotoxic T lymphocyte (CTL) population was examined from splenocytes of DC-vaccinated mice by flow cytometry. Rsad2 was induced at high levels in LPS-stimulated mDCs and mDC function was markedly attenuated under conditions of Rsad2 knockdown. Moreover, Rsad2 was necessary for mDC maturation via the IRF7-mediated signaling pathway. The importance of Rsad2 was confirmed in an Rsad2 knockdown lung metastasis mouse model in which mDCs lost their antitumor efficacy. Data on the CTL population further supported the results as above. Taken together, Rsad2 was an obvious and specific marker necessary for DC maturation and these findings will be clearly helpful for further understanding of DC biology.
\end{abstract}

\section{Introduction}

Dendritic cells (DCs) are the most potent professional antigen presenting cells and have a central role in maintaining immune homeostasis ${ }^{1}$. DCs are not only important for induction of primary immune responses, but may also have a pivotal role in priming of $\mathrm{T}$ cell-mediated immune response ${ }^{2}$. Immature DCs (imDCs) capture antigens via pattern-recognition receptors and present them to naive $\mathrm{T}$ cells as peptides bound to both major histocompatibility complex (MHC) class I and II

\footnotetext{
Correspondence: D-S. Lim (dslim@cha.ac.kr)

'Department of Biotechnology, CHA University, 335 Pangyo-ro, Bundang-gu, Seongnam, Gyeonggi-do 13488, Republic of Korea

${ }^{2}$ Pharos Vaccine Inc., 545 Dunchon-daero, Jungwon-gu, Seongnam, Gyeonggido 13215, Republic of Korea

Full list of author information is available at the end of the article.

These authors contributed equally: Ji-Su Jang, Jun-Ho Lee, Nam-Chul Jung

Edited by H.-U. Simon
}

molecules; this induces DC maturation and activates $\mathrm{T}$ cells. DC maturation cannot be defined simply by measuring a few parameters such as export of MHC class II molecules to the plasma membrane, cytokine secretion, and upregulation of co-stimulatory molecules; this is because all of these can be induced by infectious agents and inflammatory molecules ${ }^{3}$.

Although the powerful antitumor properties of mature (m)DCs have been exploited in several clinical settings, the precise underlying mechanisms and specific markers expressed by these cells remain unclear. In a previous study, we used gene profiling to identify many genes expressed by $\mathrm{mDCs}^{4}$. The results suggested that the Rsad2 (radical S-adenosyl methionine domain containing 2) gene is particularly overexpressed on $\mathrm{mDCs}$ rather than

\section{(c) The Author(s) 2018}

(c) (i) Open Access This article is licensed under a Creative Commons Attribution 4.0 International License, which permits use, sharing, adaptation, distribution and reproduction c. in any medium or format, as long as you give appropriate credit to the original author(s) and the source, provide a link to the Creative Commons license, and indicate if changes were made. The images or other third party material in this article are included in the article's Creative Commons license, unless indicated otherwise in a credit line to the material. If material is not included in the article's Creative Commons license and your intended use is not permitted by statutory regulation or exceeds the permitted use, you will need to obtain permission directly from the copyright holder. To view a copy of this license, visit http://creativecommons.org/licenses/by/4.0/. 
other DC subsets; therefore, we investigated its function in DC maturation and DC-mediated immune responses.

Rsad2 is an interferon-stimulated gene involved in innate immunity and as such is mainly responsible for antiviral responses. It is identical to cig5 (cytomegalovirus-inducible gene 5) and viperin (virus inhibitory protein, endoplasmic reticulum-associated, interferoninducible), which have received much attention recently ${ }^{5}$. Some studies have shown that the interferon-stimulated gene (ISG) viperin is physically associated with hepatitis C virus (HCV). Moreover, defining the precise mechanism of action of ISG viperin that is associated blocking of the $\mathrm{HCV}$ replication may present novel therapeutic strategies for $\mathrm{HCV}^{6}$. Rsad2 was first cloned from interferon-treated human macrophages, and is upregulated by viruses, oligonucleotides such as lipopolysaccharide (LPS) and poly I: $\mathrm{C}$, and type I interferons ${ }^{7-10}$. Viperin localizes to the endoplasmic reticulum and lipid droplets (which have an important role in the replication cycle of many viruses), where it inhibits replication of some DNA and RNA viruses $^{11,12}$. TLR3 and TLR4 receptors recognize extracellular dsRNA and LPS, respectively, and induce IRF3dependent and IRF7-dependent production of type I interferons (IFN-I; IFN- $\alpha$ and $-\beta$ ) on DCs. Rsad 2 also mediates its antiviral function by regulating the TLR7 and TLR9-IRAK1 signaling axis in plasmacytoid DCs ${ }^{13}$.

Although IFN-I are some of the earliest and most potent cytokines released in response to infection, they have an essential role in adaptive immune responses by promoting NK cell or $\mathrm{CD}^{+} \mathrm{T}$ cell antiviral cytotoxic activity, either directly or by activating conventional dendritic cells $(\mathrm{cDCs})^{14-17}$. There is some evidence that DC-secreted IFN-I act in an autocrine manner to promote survival of DC precursors and stimulate expression of IFN-I-induced genes in response to pathogen-associated signals ${ }^{18-20}$.

The mechanism underlying Rsad2-mediated adaptive immune responses is unclear. Rsad2 is expressed at high levels in mDCs in response to a wide range of viruses and microbial products such as LPS, suggesting that Rsad2 is an important component of adaptive $\mathrm{T}$ cell responses as well as innate immune responses to diverse pathogens. Thus, Rsad2 has a strong effect on the ability of mDCs to prime antigen-specific effector $\mathrm{T}$ cells. Although our understanding of DC biology is still in its infancy, we are now beginning to use DC-based immunotherapy protocols to elicit immunity responses against cancers and infectious diseases. The aims of this study were to find the role of Rsad2 in DC biology and rationale for $\mathrm{mDC}$ specific immune regulation.

\section{Results}

\section{Characterization of bone marrow-derived DCs}

A previous report shows that Rsad2 is required for mDC maturation; ${ }^{4}$ therefore, we first examined immune responses induced by $\mathrm{mDCs}$ in vitro. Cultured DCs were generated and divided in two functionally and phenotypically distinct types: immature and mature. First, mouse bone marrow-derived monocytes were cultured with GMCSF and IL-4 for 8 days to generate imDCs. Incubation of these imDCs for $24 \mathrm{~h}$ with LPS $(1 \mu \mathrm{g} / \mathrm{ml})$ led to DC maturation and significant upregulation of cell surface CD40, CD54, CD80, CD86, and MHC class II (Supplementary Fig. 1a). Although imDCs are skillful at endocytosis, they express relatively low levels of surface MHC class II product and co-stimulatory molecules compared with mDCs (e.g., CD86). The mean fluorescence intensity plots show the difference in surface marker expression between imDCs and mDCs (Supplementary Fig. 1a). Cytokine secretion is a mechanism by which DCs regulate immune responses; therefore, we next examined the cytokine profile of DCs. imDCs produced lower levels of pro-inflammatory cytokines (IL-1 $\beta$, IL-6, TNF- $\alpha$, and IL12p70) than mDCs (Supplementary Fig. 1b). Analysis of FITC-conjugated dextran uptake revealed that mDCs showed less phagocytic activity than imDCs (Supplementary Fig. 1c).

We next conducted a series of functional co-culture experiments to investigate the effect of mDCs on $\mathrm{T}$ cell proliferation and polarization. To determine whether $\mathrm{mDCs}$ promote homeostatic $\mathrm{T}$ cell proliferation more efficiently than imDCs, CFSE-labeled naive $\mathrm{T}$ cells were co-cultured with purified DC subsets for $72 \mathrm{~h}$. As shown in Supplementary Fig. 1d, mDCs-stimulated T cell proliferation to a greater extent than imDCs. Furthermore, mDCs efficiently induced Th1-mediated immune responses in co-culture experiments (Supplementary Fig. 1e). As previously reported ${ }^{21}$, when co-cultured with mDCs as stimulators, $\mathrm{CD} 4^{+} \mathrm{T}$ cells produce Th1 cytokines (IFN- $\gamma$ ) rather than the Th2 cytokines (IL-4); imDCs were clearly less able to stimulate Th1 cell responses (Supplementary Fig. 1e). Additionally, mDCs were significantly better than imDCs at inducing $\mathrm{T}$ cell-mediated production of IFN- $\gamma$ and IL-17A (Supplementary Fig. 1f). However, no Th2 cytokines (IL-4) were detected. These results indicate that $\mathrm{mDCs}$ induce $\mathrm{T}$ cell-mediated adaptive immune responses.

\section{Expression of Rsad 2 in $\mathrm{mDCs}$}

To determine whether mDCs express Rsad2, we cultured mouse bone marrow-derived monocytes with GMCSF and IL- 4 for 8 days to generate imDCs. These cells were treated with LPS to generate mDCs and mRNA was extracted for reverse transcriptase (RT)-PCR and realtime PCR. The results showed that Rsad2 was expressed by $\mathrm{mDCs}$ (Fig. 1a, b, respectively). In addition, western blotting showed that mDCs expressed more Rsad2 protein than imDCs (Fig. 1c). Next, we performed an immunofluorescence assay to examine localization of 
a

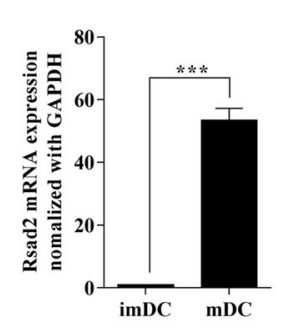

Rsad2

GAPDH

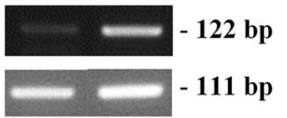

c

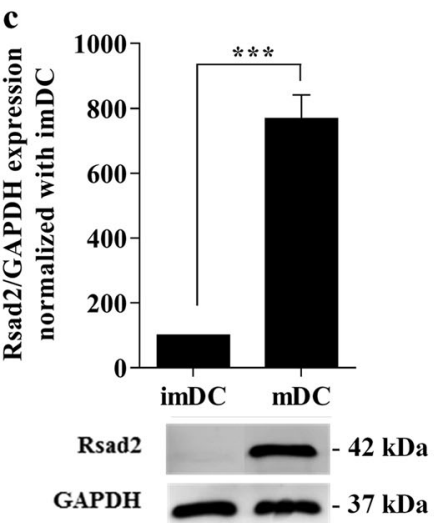

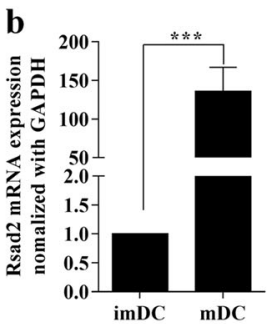

d DAPI

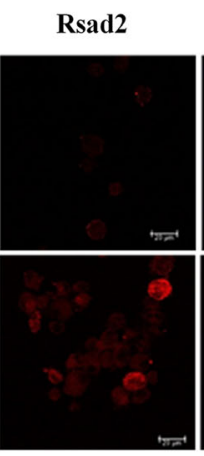

Merged

imDC

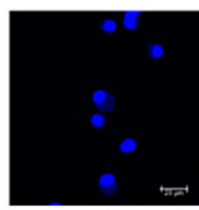

mDC

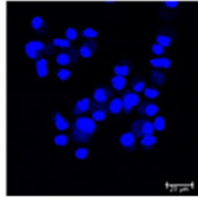

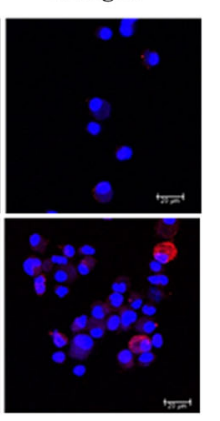

Fig. 1 Mature dendritic cells (mDCs) express high levels of Rsad2. a PCR to detect Rsad2 expression in DCs. At day 8, DCs were cultured with LPS $(1 \mu \mathrm{g} / \mathrm{ml}), \mathrm{KLH}(10 \mu \mathrm{g} / \mathrm{ml})$ for mDC, or control medium only for imDC. Values were normalized to GAPDH expression. RT-PCR analysis was repeated three times and data expressed as the mean \pm SEM. $\mathbf{b}$ qRT-PCR assay to detect Rsad2 mRNA expression. The bar graphs show the mean fluorescence intensity, expressed as the mean \pm SEM ( $n=10$ independent DC preparations). c Western blot analysis of Rsad2 expression. Data are representative of at least three independent experiments. $\mathbf{d}$ Immunofluorescence analysis of endogenous Rsad2 (red) expression in DCs. Nuclei are stained with DAPI (blue). ${ }^{*} P<0.05,{ }^{* *} P<0.01$, and ${ }^{* * *} P<0.001$, compared with imDC

Rsad2 in mDCs. As shown in Fig. 1d, Rsad2 was detected in the cytoplasmic of mDCs but not in imDCs. These results imply that the Rsad2 is only endogenously expressed by LPS-stimulated DCs.

\section{Rsad2 knockdown mDCs show functional defects}

To confirm whether the Rsad2 knockdown affects the function of mDCs, we transfected cells with Rsad2 siRNA (100 nM for $48 \mathrm{~h}$ ) or scramble siRNA. Our preliminary studies showed that transfecting Rsad2-specific siRNA into DCs yielded the greatest protein knockdown at $48 \mathrm{~h}$. The efficiency of siRNA-mediated Rsad2 knockdown was confirmed by qPCR and western blotting (Fig. 2a, b). Although the DCs had been stimulated with LPS for $24 \mathrm{~h}$, knockdown of Rsad 2 led to a significant reduction in their secretion of pro-inflammatory cytokines (Fig. 2c). However, similar levels of cell surface marker expression were detected on mDCs and Rsad 2 knockdown mDCs. Indeed, Rsad2 knockdown mDCs and mDCs showed similar expression of CD80 and CD86, as well as MHC class II (or slightly higher expression in mDCs) (Fig. 2d).
Next, to clarify whether Rsad2 knockdown mDCs lose the ability to stimulate $\mathrm{T}$ cells, we co-cultured Rsad2 knockdown cells with $\mathrm{T}$ cells. As shown in Fig. 2e, T cell proliferation in the presence of DCs transfected with Rsad 2 siRNA was markedly reduced at $72 \mathrm{~h}$. In addition, the Th1 T cell population was markedly depleted at $72 \mathrm{~h}$ (Fig. 2f). Parallel assays revealed that $\mathrm{T}$ cell-mediated secretion of IFN- $\gamma$ was also reduced by $80 \%$ at $72 \mathrm{~h}$ (a similar propensity in the IL-17A secretion) (Fig. 2g). Taken together, these results clearly demonstrate that Rsad2 has a marked effect on the interaction between mDCs and $\mathrm{T}$ cells.

\section{Rsad2 is required for IRF7-mediated type I IFNs secretion by $\mathrm{mDCs}$}

As shown above, knockdown of Rsad2 abrogated mDCmediated immune responses. Therefore, we conducted western blot analysis to identify the signaling molecules involved. We examined phosphorylation of NF-kB, ERK, JNK, p38, and IRF7 that are essential in regulating many cellular processes including inflammation, cell stress 

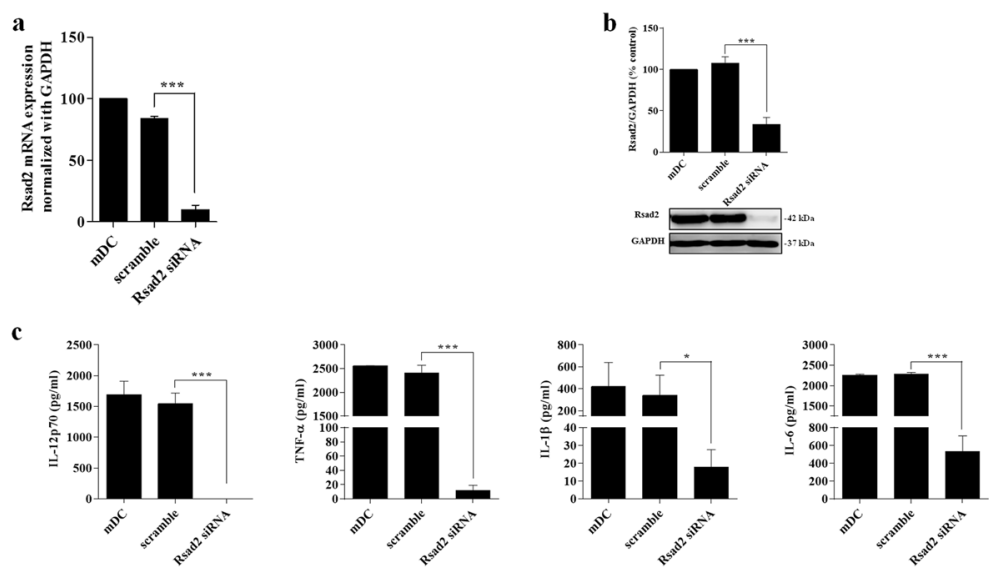

d

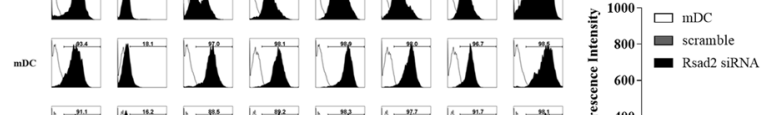

-TIITITA

IIIIIII A

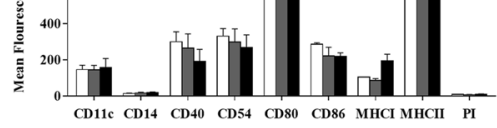

e
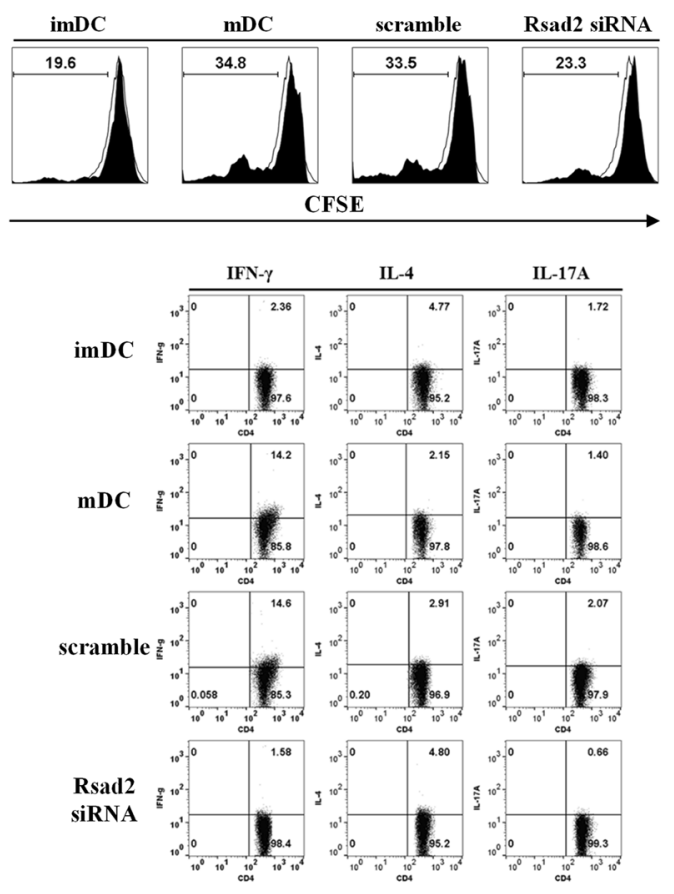

g

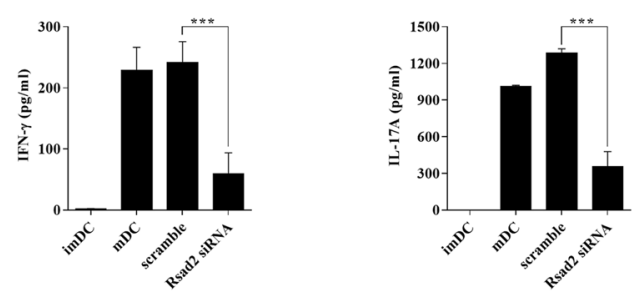

Fig. 2 (See legend on next page.) 
(see figure on previous page)

Fig. 2 Rsad2 knockdown mDCs are less efficient at stimulating T cells. a qRT-PCR assay to measure Rsad2 expression in DCs. mDCs were cultured with LPS $(1 \mu \mathrm{g} / \mathrm{ml})$ or $\mathrm{KLH}(10 \mu \mathrm{g} / \mathrm{ml})$, or in Rsad2 or scramble siRNA-transfected. Samples were normalized against GAPDH expression. The RT-PCR analysis was repeated three times and data presented as the mean \pm SEM. $\mathbf{b}$ Western blot analysis of Rsad2 knockdown. Data are representative of at least three independent experiments. c Cytokine levels in supernatants from DC cultures measured by ELISA. Data are expressed as mean \pm SEM ( $n=$ 10 independent DC preparations). d DC subsets (imDCs, mDCs, scramble-mDCs, and Rsad2 knockdown mDCs) were stained with the fluorescentlyconjugated antibodies specific for the indicated molecules and analyzed by flow cytometry. Data are presented as histograms (data are representative of ten independent DC preparations). The bar graphs show the mean fluorescence intensity, expressed as the mean \pm SEM ( $n=10$ independent DC preparations). e Each DC subset was co-cultured with CFSE-labeled CD3 ${ }^{+} \mathrm{T}$ cells and T cell proliferation measured after $72 \mathrm{~h}$. The stimulator:responder ratio was 1:10. The bar graphs show the mean fluorescence intensity, expressed as the mean \pm SEM $(n=10$ independent DC preparations). $\mathbf{f} \mathrm{T}$ cell subpopulations were analyzed by flow cytometry. DCs were co-cultured with $\mathrm{CD} 3^{+} \mathrm{T}$ cells and the Th1 $\left(\mathrm{CD} 4^{+} \mathrm{IFN}-\gamma^{+}\right)$, Th2 $\left(\mathrm{CD} 4^{+} \mathrm{IL}-4^{+}\right)$, and Th17 $\left(\mathrm{CD}^{+}{ }^{+} \mathrm{LL}-17 \mathrm{~A}^{+}\right)$cell population were detected by flow cytometry. $\mathbf{g}$ Measurement of Th1/Th17 cytokines in culture supernatants from DCs $/ T$ cell co-cultures by ELISA. Data are expressed as mean \pm SEM ( $n=10$ independent DC preparations). ${ }^{*} P<0.05$; ${ }^{* *} P<0.01$; ${ }^{* * *} P<0.001$, compared with imDC

response, cell differentiation, cell division, cell proliferation, metabolism, motility, and apoptosis. The basal levels of NF- $\mathrm{kB}$ and MAPK in freshly isolated mDCs and Rsad2 knockdown mDCs were similar (Fig. 3a). However, Rsad2 knockdown mDCs showed a marked defect in expression of phosphorylated IRF7 (Fig. 3a). Therefore, we conducted immunofluorescence analysis to visually show an association with IRF7 in mDCs. As shown in Fig. 3b, mDCs expressed Rsad2; importantly, reduced phospho-IRF7 expression was observed in Rsad2 knockdown mDCs. These results indicate that $\operatorname{Rsad} 2$ contribute to a process of DC maturation via the IRF7-mediated signaling pathway.

Given that Rsad2-mediated expression of IRF-3 and IRF-7 in macrophages contributes to IFN-I production by these cells ${ }^{22}$, we suspected that deficient Rsad2 expression in mDCs would have an effect on IFN-I secretion. Indeed, we found reduced amounts of IFN-I mRNA in Rsad2 knockdown mDCs (Fig. 3c). Consistent with this, mDCs secreted significantly more IFN-I cytokine than Rsad2 knockdown mDCs (Fig. 3d).

\section{Rsad2-mediated induction of CTL responses}

We next determined whether loss of Rsad2 affects induction of CTL and their cytotoxicity. The percentage of $\mathrm{CD}^{+} \mathrm{IFN}^{+}{ }^{+} \mathrm{T}$ lymphocytes was determined by flow cytometry. As shown in Fig. 4a, T cells co-cultured with B16F10 cell lysate-pulsed mDCs contained a higher percentage of $\mathrm{CD}^{+}{ }^{+} \mathrm{FN}-\gamma^{+} \mathrm{T}$ lymphocytes than those cocultured with B16F10 cell lysate-pulsed Rsad2 knockdown mDCs.

Next, we measured release of cytosolic LDH into the culture medium by a damaged B16F10 melanoma cell line to examine the cytotoxic activity of $\mathrm{T}$ cells co-cultured with B16-specific mDCs. B16F10 lysate-pulsed mDC-stimulated $\mathrm{T}$ cells (effector cells) were co-cultured with B16F10 melanoma cells (target cells) at effector-to-target cell ratios of 3:1, 9:1, and 27:1. As shown in Fig. 4b, B16F10 lysate-pulsed mDC-stimulated $\mathrm{T}$ cells induced greater cytotoxic responses than B16F10 lysate-pulsed
Rsad2 knockdown mDCs-stimulated T cells. However, co-culture with another cancer cell line (YAC-1) did not affect CTL cytotoxicity. Neither B16F10 melanoma cells nor naive $\mathrm{T}$ cells cultured alone in control medium (negative controls) released any LDH at $4 \mathrm{~h}$. These results suggest that high-activity CTLs were induced by coculture with B16F10-pulsed mDCs but not by co-culture with Rsad2 knockdown cells. To this end, key concepts of Rsad2-mediated phosphorylation of IRF7 in the mDCs, resulting antigen-specific $\mathrm{T}$ cell induction.

\section{B16F10 lysate-pulsed mDCs show stronger antitumor effects in vivo than lysate-pulsed Rsad2 knockdown mDCs}

Finally, to examine antitumor responses in vivo, B16F10 tumor-bearing mice were immunized with $\mathrm{mDCs}$ or Rsad2 knockdown mDCs and survival evaluated. Mice bearing B16F10 tumors were vaccinated with $\mathrm{mDCs}$ on days 3 and 10 after initial tumor cell injection (Fig. 5a). As shown in Fig. 5b, mice treated with B16F10-pulsed mDCs showed little or no growth of B16F10 tumors in the lungs; by contrast, mice treated with $\mathrm{PBS}$ alone or with Rsad2 knockdown mDCs harbored many B16F10 tumors.

Next, to compare differences in the in vivo immune responses in mice vaccinated with B16F10-pulsed mDCs or Rsad2 knockdown mDCs, we analyzed IFN- $\gamma$ producing $\mathrm{CD} 8 \mathrm{~T}$ cell populations. Figure $5 \mathrm{c}$ shows that vaccination with B16F10-pulsed Rsad2 knockdown mDCs induced more reduced $\mathrm{CD} 8 \mathrm{~T}$ cell populations than lysate-pulsed mDCs. Moreover, vaccination with B16F10pulsed $\mathrm{mDCs}$ resulted in potent stimulation of IFN- $\gamma$ secreting $\mathrm{CD}^{+}{ }^{+} \mathrm{T}$ cells (Fig. $5 \mathrm{~d}$ ). The results revealed that i.v. immunization with B16F10 cell lysate-pulsed mDCs protected mice against formation of B16F10 melanomas. These results imply that $R s a d 2$-expressing mDCs induce a potent antitumor response.

\section{Discussion}

One of the most critical features of DC biology is functional maturation, which influences the fundamental properties of an immune response ${ }^{1}$. Two major types of 


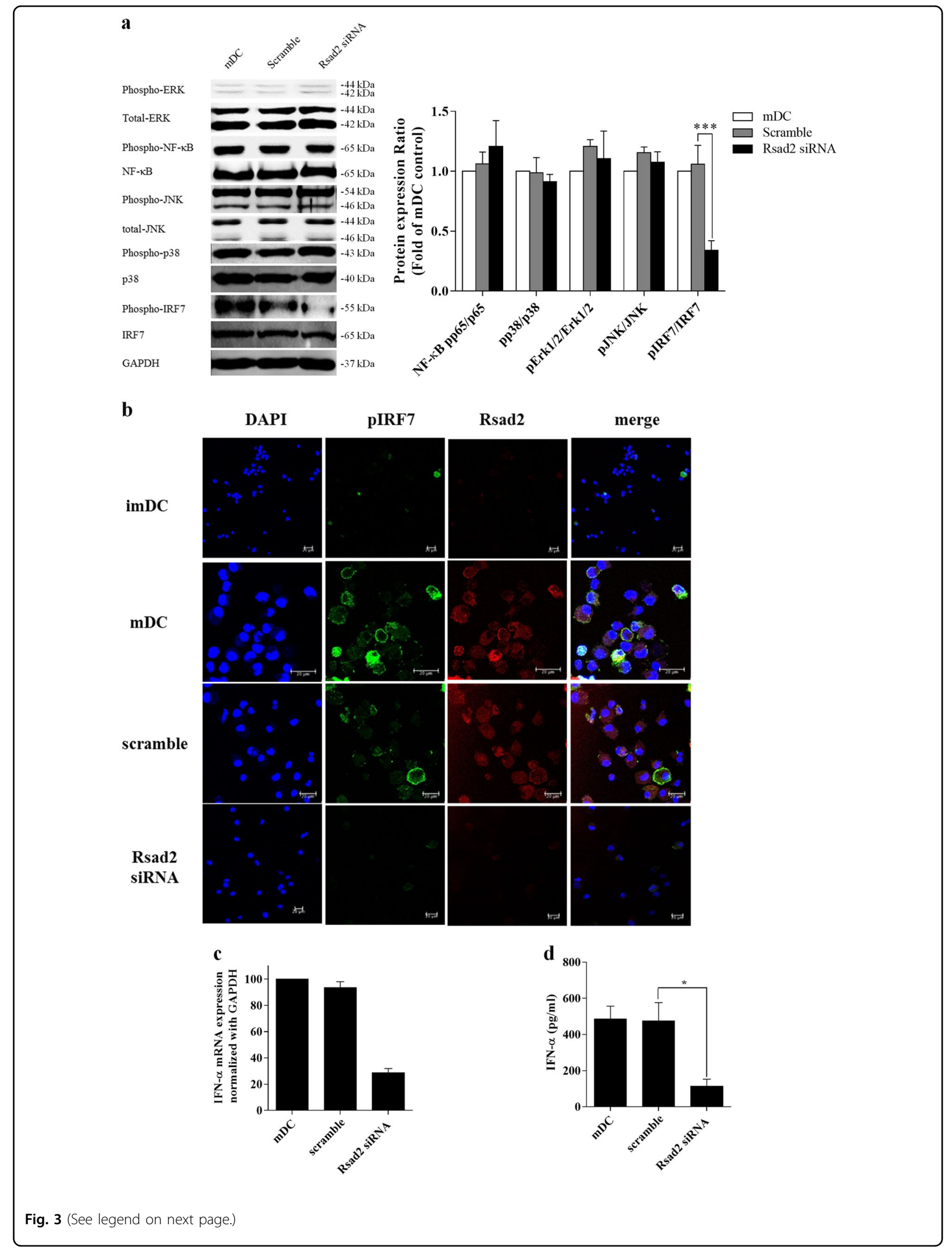


(see figure on previous page)

Fig. 3 Rsad2 is required for IRF7-mediated secretion of type I IFN by mDCs. a Western blot analysis of MAPKs, NF-KBp65, and IRF7. Data are representative of at least three independent experiments. b Immunofluorescence analysis of endogenous Rsad2 (red) and p-IRF7 (green) expression in DCs. Nuclei are stained with DAPI (blue). c qRT-PCR assay measuring type I IFN expression by each DC subset. Sample data were normalized against GAPDH expression. RT-PCR analysis was repeated three times and data presented as the mean \pm SEM. $\mathbf{d}$ IFN- $\mathrm{a}$ (an IFN-I cytokine) levels in the supernatant of each DC subset measured by ELISA. Data are expressed as mean \pm SEM ( $n=10$ independent DC preparations). ${ }^{*} P<0.05$, ${ }^{* *} P<0.01$, and ${ }^{* *} P<0.001$

DC are present under inflammatory conditions: imDCs and mDCs. These DC subsets express different levels/types of pro-inflammatory cytokines and show differing abilities to stimulate $\mathrm{T}$ cell proliferation and IFN- $\gamma$-mediated CTL responses $^{3}$. Although these different phenotypes provide clues as to cellular function, the different DC subsets share some features under inflammatory conditions. Therefore, there is a need to identify reliable DC markers that will enable identification of different DC subsets ${ }^{21,23}$.

Among innate inflammatory cytokines, IFN-I are considered major players that link innate and adaptive immunity by activating DCs. IFN-I promote expression of co-stimulatory molecules, and IFN-I-treated DCs prime $\mathrm{T}$ cells more effectively in vitro ${ }^{17,24-26}$. Rsad2 has a dual function: direct suppression of viral replication and facilitation of TLR7-mediated and TLR9-mediated production of IFN-I ${ }^{27}$. A study based on human monocytederived DCs found that Rsad2 is expressed at higher levels in type 2 DCs, which have the ability to induce differentiation of naive $\mathrm{T}$ cells into Th2 cells by activating the NF- $\mathrm{kB}$ and AP-1 signaling pathways ${ }^{28}$. Additionally, Rsad2 shows direct and indirect antiviral activity against various viruses and is induced directly in myeloid DCs by IRF-3-mediated and 7-mediated production of type 1 IFN $^{11,12,22}$.

Here, we show that expression of Rsad2, a typical interferon-stimulated gene (ISG), was upregulated in fully matured DCs. To understand how Rsad2 induces mDC function, we generated Rsad2 knockdown cells. Rsad2 knockdown mDCs lost the ability to promote proinflammatory cytokine production and induce $\mathrm{T}$ cell proliferation. Next, we confirmed that phosphorylation of IRF7 has a role in Rsad2-mediated mDC function. Overall, the results provide insight into the role of Rsad2 in $\mathrm{mDC}$ function and IRF7-mediated production of IFN-I. Thus, Rsad2 is not only involved in antiviral innate immune responses, but is also a powerful stimulator of adaptive immune response mediated via mDCs. Furthermore, it is
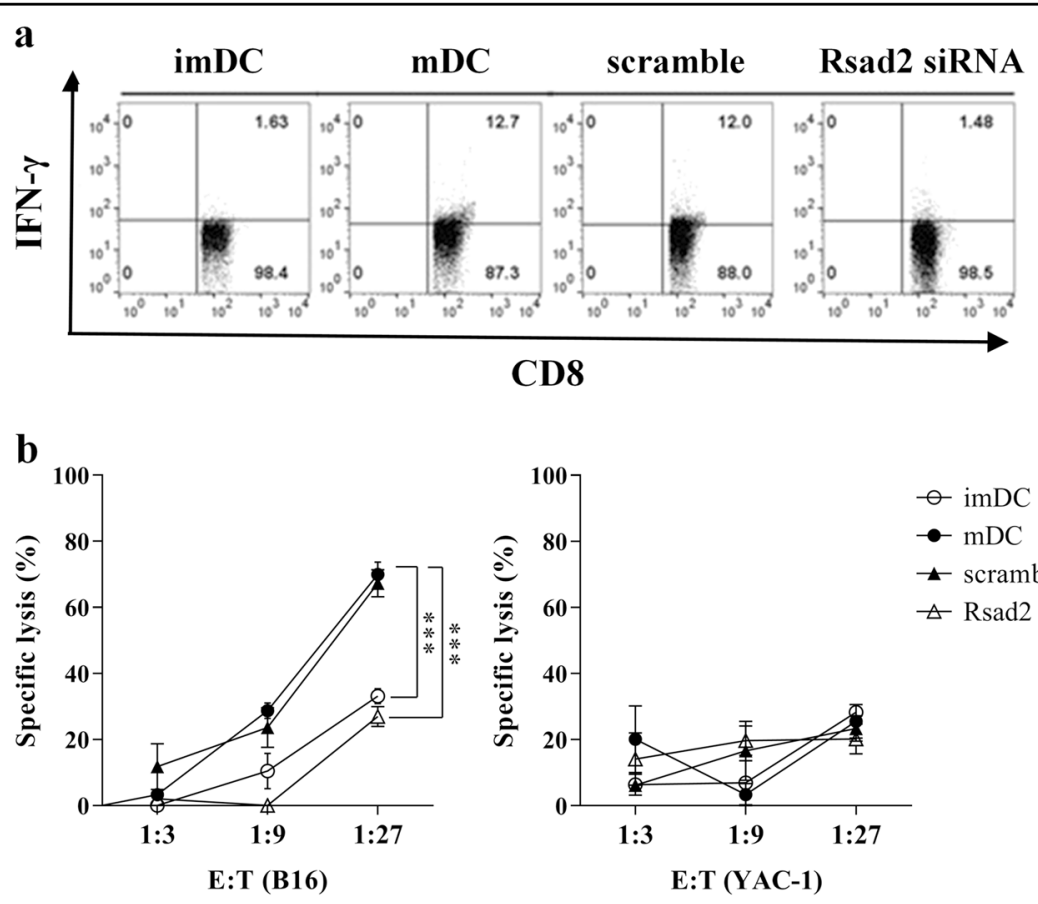

Fig. 4 Rsad2-mediated induction of CTL responses. a Each DC subset was co-cultured with $\mathrm{CD}^{+}{ }^{+}$cells for 14 days in the presence of $\mathrm{IL}-2(100 \mathrm{U} / \mathrm{ml})$. The stimulator:responder ratio was 1:10. The B16F10-specific IFN- $\gamma^{+} \mathrm{CD} 8^{+} \mathrm{T}$ cell population was then analyzed by flow cytometry. b LDH assay to measure primary B16F10-specific CTL responses to each DC subset. The line graphs show the mean fluorescence intensity, expressed as the mean \pm SEM ( $n=5$ independent preparations) 


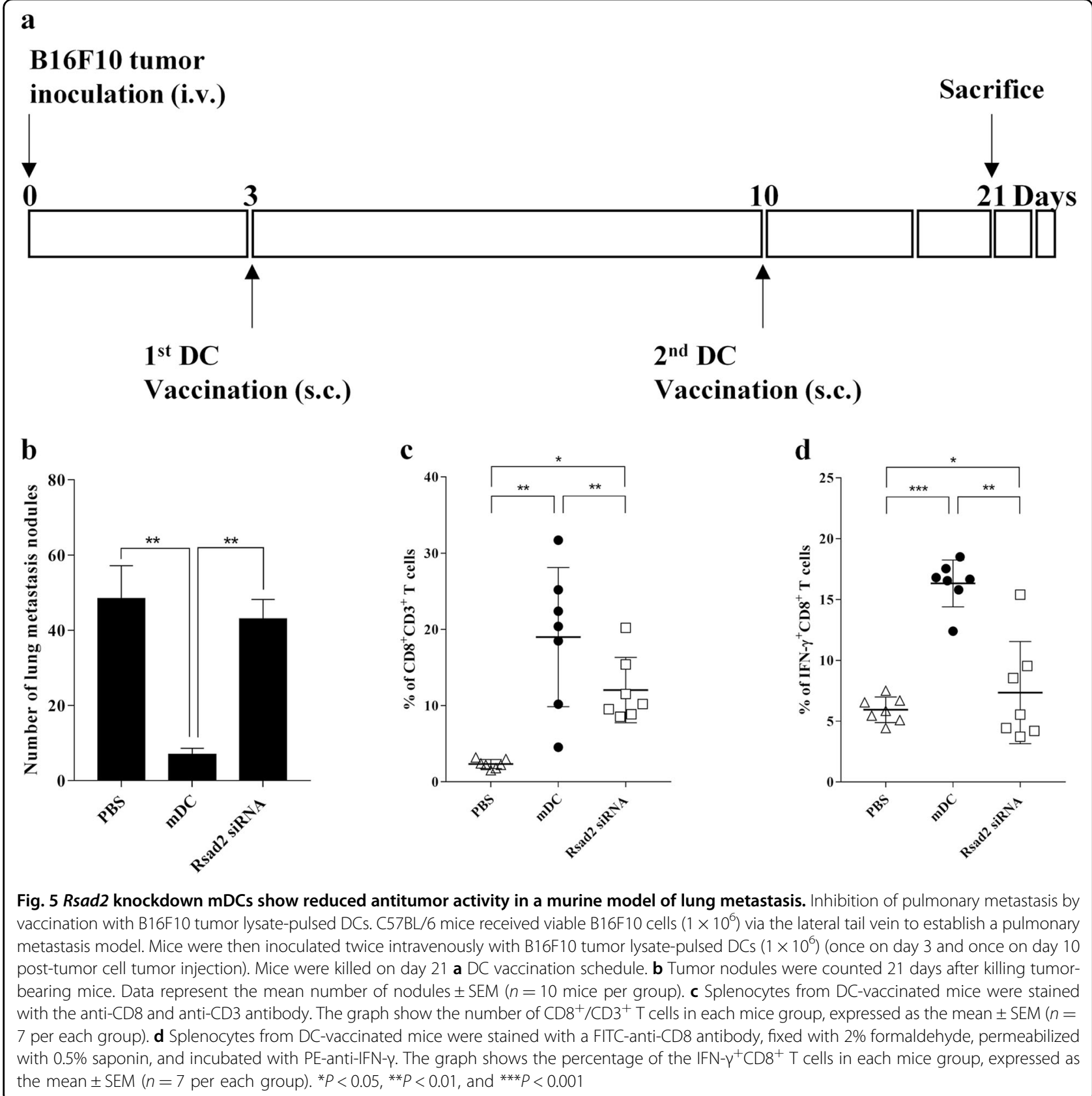

required to confirm reproducibility of these results by using human DCs. Rsad2 is a specific marker for DC maturation, overexpression of which may lead to development of powerful cell-based immunotherapies.

\section{Materials and methods}

\section{Ethical approval}

All protocols involving the use of animals were approved by the Institutional Animal Care and Use Committee of CHA University (Project No. IACUC160095), and all experiments were carried out in accordance with these approved protocols.

\section{Mice}

Male C57BL/6 mice (6-8 weeks-of-age, each weighing $14-16 \mathrm{~g}$ ) were purchased from Orient Bio Inc. (Gyeonggi, Republic of Korea). All mice were housed in a temperature- and humidity-controlled room under a $12 \mathrm{~h} / 12 \mathrm{~h}$ light/dark cycle.

\section{Generation of DCs}

DCs were generated from bone marrow progenitors obtained from 6-week-old C57BL/6 mice, as described previously ${ }^{4}$. Briefly, bone marrow single cell suspensions were prepared as described ${ }^{4}$, washed with PBS (Lonza, 
Basel, Switzerland), and cultured in RPMI 1640 (Lonza) supplemented with 10\% FBS (Corning, NY, USA), $1 \times$ antibiotics (Gibco, Life Technologies, Grand Island, NY, USA), $20 \mathrm{ng} / \mathrm{ml}$ recombinant mouse (rm) GM-CSF (JWCreaGene, Gyeonggi, Korea) and $2 \mathrm{ng} / \mathrm{ml} \mathrm{rmIL-4}$ (JWCreaGene). Cells were cultured at $37{ }^{\circ} \mathrm{C}$ under $5 \%$ $\mathrm{CO}_{2}$. After 3 days, another $10 \mathrm{ml}$ of complete medium containing GM-CSF was added to each dish. On day 6, $50 \%$ of the culture medium was replaced with fresh medium containing the same concentrations of GM-CSF and IL-4. On day 8, the non-adherent cells were harvested and mDCs treated for $24 \mathrm{~h}$ with $1 \mu \mathrm{g} / \mathrm{ml}$ LPS (Sigma-Aldrich, St Louis, MO, USA) or $10 \mu \mathrm{g} / \mathrm{ml}$ Keyhole Limpet Hemocyanin (KLH) (Sigma-Aldrich). For the lactate dehydrogenase (LDH) release assays and in vivo studies, tumor cell lysates were used as a source of antigen.

\section{Flow cytometry analysis}

Phenotypic analysis was performed by direct immunofluorescence staining of DC cell surface markers. Cells (in PBS) were stained with the following antibodies at $4{ }^{\circ} \mathrm{C}$ for 20 min: Phycoerythrin (PE)-conjugated antiCD11c (Clone N418); Fluorescein isothiocyanate (FITC)-conjugated anti-CD14 (Clone Sa14-2); PEconjugated anti-CD40 (Clone 3/23); FITC-conjugated anti-CD54 (Clone 3E2); PE-conjugated anti-CD80 (Clone 16-10A1); FITC-conjugated anti-CD86 (Clone PO3); FITC-conjugated anti-MHC I (Clone KH95), or PE-conjugated anti-MHC II (Clone 2G9) (all antibodies were purchased from BD Pharmingen, San Diego, CA, USA), plus appropriate isotype controls. Cells were then analyzed using a 3-color FACSCalibur cytometer (BD Biosciences, Mountain View, CA). Data were collected from 10,000 events and analyzed using FlowJo10 software (BD Biosciences).

\section{Cytokine measurement}

The concentrations of interleukin (IL)-1 $\beta$, IL-6, IL-10, IL-12p70, and IL-4 secreted by lymphocytes or DCs were measured using commercially available ELISA kits (all kits were purchase from BioLegend, CA, USA) in accordance with the manufacturer's instructions.

\section{Dextran uptake assay}

Phagocytic activity was examined as previously described $^{29}$. Briefly, immature DCs and $\mathrm{mDCs}$ were incubated with FITC-conjugated dextran $(1 \mathrm{mg} / \mathrm{ml}$; Sigma-Aldrich) in culture medium for $1 \mathrm{~h}$ at $37^{\circ} \mathrm{C}$. Next, cells were washed with $\mathrm{PBS}$ prior to analysis by flow cytometry (FACSCalibur ${ }^{\mathrm{mm}}$; BD Biosciences). Cells positive for FITC (detected by fluorescence detector 1 ) were identified as cells that had engulfed dextran.

\section{Quantitative real-time PCR (qRT-PCR)}

RNA was isolated using LABOzol, according to the manufacturer's instructions (Invitrogen, California, USA). RNA $(1 \mu \mathrm{g})$ was then used to synthesize cDNA using a LaboPass cDNA synthesis kit (Cosmogenetech, Seoul, Korea). cDNA samples were subjected to real-time PCR analyses with Rsad2 primers and a SensiFAST SYBR HiROX-kit (Bioline, London, UK) using the real-time quantitative RT-PCR (Applied Biosystems, Foster City, CA, USA). The primer sequences were as follow: Rsad 2 forward, $5^{\prime}$-GGTGCCTGAATCTAACCAGAAG-3' and Rsad2 reverse, 5'-CCACGCCAACATCCAGAATA-3'; GAPDH forward, 5'-AACAGCAACTCCCACTCTTC-3' and reverse, 5'-CCTGTTGCTGTAGCCGYATT-3'. The normalized value for Rsad 2 mRNA expression were calculated as the relative quantity of Rsad2 divided by the relative quantity of $G A P D H$. All samples were run in triplicate.

\section{Western blot analysis}

Whole cell lysates for western blot were prepared as described $^{30}$. Protein concentrations of whole cell lysates were measured using a Bradford assay kit (SigmaAldrich). Equal amounts of protein were loaded into the wells of a SDS-PAGE gel and the separated proteins transferred to PVDF membranes (Thermo Scientific, Hudson, NH, USA). The membranes were blocked with $10 \%(\mathrm{w} / \mathrm{v})$ skim milk in PBST and then incubated with primary antibodies (anti-Rsad2 (Abcam, Cambridge, UK), p-Erk1/2, Erk1/2, p-NF-кBp65, NF-кBp65, p-JNK, JNK, p-p38, p38, p-IRF7, IRF7, and GAPDH; all diluted 1:1000; Cell Signaling Technologies, Danvers, MA, USA) overnight at $4{ }^{\circ} \mathrm{C}$. The membranes were then washed with PBST and incubated with an HRP-conjugated rabbit antimouse IgG and mouse anti-goat secondary antibody (diluted 1:2000; Cell Signaling Technologies) for $2 \mathrm{~h}$ at room temperature. The membrane was then exposed to ECL reagents (Thermo Scientific) and the resulting signals detected using a Luminescent image analyzer (LAS4000; Fuji Film, Tokyo, Japan).

\section{RNA interference}

For delivery of small interfering RNAs (Rsad2 siRNAs; sense: 5'-GCAGAAAGAUUUCUUAUAA-3'; antisense: $5^{\prime}$-UUAUAAGAAAUCUUUCUGC- $3^{\prime}$ ), DCs were plated into each well of a 6-well plate (SPL Life Sciences, Gyeonggi, Republic of Korea) and transfected with siRNA duplexes using the Lipofectamine 3000 transfection reagent (Invitrogen), according to the manufacturer's instructions. Briefly, cells were cultured for $4 \mathrm{~h}$ with the transfection mixture in OptiMEM medium (Invitrogen). Next, a volume of medium equal to that of the Lipofectamine-100 nM Rsad2 siRNA mixture was added. 
Four hours later, the cells were plated into 24-well dishes and stimulated with $1 \mu \mathrm{g} / \mathrm{ml}$ LPS. The next day, the cells were subjected to a second round of transfection to increase siRNA-mediated gene suppression. At $24 \mathrm{~h}$ postsecond transfection, the cultures were harvested and prepared for further analysis.

\section{CFSE proliferation assay}

Splenocytes were isolated from wild type C57BL/6 mice spleen and disaggregated in RPMI 1640 medium. $\mathrm{CD}^{+} \mathrm{T}$ cells were isolated by passing the splenocytes through a nylon wool (Polysciences Inc., Warrington, PA, USA) column. Nylon-wool column-purified T cells from spleens were labeled using CFSE labeling kits (Molecular Probes, Oregon, USA) according to the manufacturer's instructions. Cells were then co-cultured with DCs at a DC:T cell ratio of 1:10. CFSE-labeled purified $\mathrm{CD}^{+} \mathrm{T}$ cells $\left(1 \times 10^{6}\right.$ cells $\left./ \mathrm{ml}\right)$ were used as responders and imDCs or $\mathrm{mDCs}\left(1 \times 10^{6}\right.$ cells $\left./ \mathrm{ml}\right)$ were used as stimulators. Cells were co-cultured at $37^{\circ} \mathrm{C}$ for $72 \mathrm{~h}$ in $2 \mathrm{ml}$ of RPMI 1640 supplemented with 10\% FBS.

\section{Generation of CTLs}

DCs loaded with B16F10 cell lysates were sorted and used as stimulatory cells, while $\mathrm{CD}^{+}{ }^{+} \mathrm{T}$ cells in a final volume of $1 \mathrm{ml}$. The RPMI 1640 media was supplemented with $10 \% \mathrm{FBS}$ and IL-2 $(10 \mathrm{IU} / \mathrm{ml}$ at weeks 2 and 3 of culture). $T$ cells were re-stimulated weekly (for an additional 2 weeks) with B16F10 lysates loaded or unloaded DCs. Six days after the last stimulation, $\mathrm{T}$ cells were harvested and cytotoxic activity and the capacity for IFN- $\gamma$ release tested.

\section{Preparation of tumor cell lysates}

Confluent cultures of B16F10 or YAC-1 cells were incubated for $10 \mathrm{~min}$ with $0.25 \%$ trypsin-EDTA solution (Gibco, Life Technologies), carefully detached, washed twice in PBS, and resuspended at a density of $5 \times 10^{6} / \mathrm{ml}$ in serum-free DMEM (Lonza). The cell suspensions were frozen at $-80^{\circ} \mathrm{C}$, and lysed by repeated (four times) freeze-thaw cycles. To remove crude debris, the lysate was centrifuged for $10 \mathrm{~min}$ at $300 \times g$. The supernatant was then collected and passed through a $0.2-\mu \mathrm{m}$ filter (SigmaAldrich). The protein concentration of the lysate was measured in a commercial assay (Bio-Rad, Munich, Germany).

\section{Cytotoxicity assay}

The CytoToxw96 Non-Radioactive Assay kit (Promega, WI, USA) was used to measure $\mathrm{T}$ cell-mediated cytotoxicity. CTLs (effector cells) and B16F10 tumor cells (target cells) were co-cultured in round bottom 96-well plates for $4 \mathrm{~h}$ in $37^{\circ} \mathrm{C} / 5 \% \mathrm{CO}_{2}$. Next, lysis solution $(20 \mu \mathrm{l}$; $10 \mathrm{ml} / 100 \mathrm{ml}$ ) was added to four wells and incubated for
$45 \mathrm{~min}$. These wells acted as positive controls (maximum release of LDH). Next, $50 \mu \mathrm{l}$ of supernatant from each well was placed in a new 96-well plate and $50 \mu \mathrm{l}$ of reconstituted substrate mix added. The plates were then kept in a dark room at room temperature for $30 \mathrm{~min}$. Stop solution $(50 \mu \mathrm{l})$ was then added to each well for $1 \mathrm{~h}$. Absorbance $(490 \mathrm{~nm})$ was recorded using a Dynatech MR 4000 plate reader (Dyantech laboratories Billingshurst, UK). Each experiment was repeated six times. Cytotoxicity was evaluated with the CytoTox96 Non-Radioactive Cytotoxicity Assay kit (Promega) following the manufacturer's protocol; the colorimetric assay quantifies lactate dehydrogenase (LDH) activity released from the cytosol of damaged target cells into the supernatants.

\section{Confocal microscopy analysis}

Confocal analysis was conducted as previously described $^{24}$. After fixation for $10 \mathrm{~min}$ in $4 \%$ paraformaldehyde in PBS, cells were washed in 1\% Tween20/PBS and permeabilized with $0.1 \%$ Triton-X 100 (Sigma-Aldrich) in PBS. Cells were then incubated in 5\% NGS in room temperature for blocking. After washing with $1 \%$ Tween 20 in PBS, cells were incubated for $24 \mathrm{~h}$ at $4{ }^{\circ} \mathrm{C}$ with an anti-Rsad2 antibody (Abcam). After further washing in $1 \%$ Tween20 in PBS, cells were stained for $2 \mathrm{~h}$ at room temperature with a secondary antibody (Alexa fluor 488 or 555-conjugated anti-mouse Ab (DAKO, Santa Clara, CA, USA)) in DAKO antibody diluent solution (DAKO). Cells were then resuspended in DAPI (DAKO). Confocal microscopy was performed using a Leica TCS-NT SP equipped with argon, krypton, and helium/neon lasers, and a spectrophotometer was used to separate the detection channels of Alexa fluor488 $(450-500 \mathrm{~nm})$ and Alexa fluor555 $(580-660 \mathrm{~nm})$.

\section{Immunization of mice and tumor challenge}

B16F10 cells were trypsinized, washed twice in $1 \times \mathrm{PBS}$ to eliminate serum proteins, and suspended in PBS (at $1 \times$ $10^{7}$ cells $/ \mathrm{ml}$ ) prior to injection. Male $\mathrm{C} 57 \mathrm{BL} / 6$ mice (7-10 weeks old) were challenged intravenously (i.v.) with $100 \mu \mathrm{l}\left(1 \times 10^{6}\right.$ cells $)$ of B16F10 cell. After 3 days, B16F10 lysate $\left(\sim 100 \mu \mathrm{g}\right.$ of proteins)-pulsed $\mathrm{mDCs}\left(1 \times 10^{6}\right.$ cells $)$ were injected into the subcutaneously (s.c.) once on day 3 and once on day 10. Mice were killed on day 21. The lungs were removed and washed for $10 \mathrm{~min}$ in Bouin's fluid (Sigma-Aldrich). The lobes of the lungs were separated and the total number of superficially visible tumor cell colonies per lung was counted. Lungs containing tumor nodules were photographed.

\section{Statistical analysis}

All assays were performed in triplicate (at least). Data are expressed as the mean \pm standard error of the mean (SEM). Data were analyzed by one-way ANOVA and a 
$P$ value $<0.05$ was considered statistically significant. All statistical analyses were performed using Prism software (GraphPad Prism v5.0; GraphPad Prism Software, San Diego, CA).

\section{Acknowledgements}

This research was supported by the Bio \& Medical Technology Development Program of the National Research Foundation (NRF) funded by the Korean government (MSIP) (No. NRF-2017M3A9C8062622).

\section{Author contributions}

J.-S.J., J.-H.L., N.-C.J., and D.-S.L. designed research; J.-S.J., S.-Y.C., S.-Y.P., and J.-Y.Y. performed research; J.-S.J., J.-H.L., N.-C.J., S.-Y.C., S.-Y.P., J.Y.Y., J.-Y.S., H.G.S., H.S.L. and D.-S.L. analyzed data; and J.-S.J., J.-H.L., and D.-S.L. wrote the paper.

\section{Author details}

'Department of Biotechnology, CHA University, 335 Pangyo-ro, Bundang-gu, Seongnam, Gyeonggi-do 13488, Republic of Korea. ${ }^{2}$ Pharos Vaccine Inc., 545 Dunchon-daero, Jungwon-gu, Seongnam, Gyeonggi-do 13215, Republic of Korea. ${ }^{3}$ Department of Radiation Cancer Sciences, Korea Institute of Radiological and Medical Sciences, 75 Nowon-ro, Nowon-gu, Seoul 01812 , Republic of Korea. ${ }^{4}$ Department of Food Science and Biotechnology of Animal Products, Sanghuh College of Life Sciences, Konkuk University, 120 Neungdong-ro, Gwangjin-gu, Seoul 05029, Republic of Korea

\section{Conflict of interest}

The authors declare that they have no conflict of interest.

\section{Publisher's note}

Springer Nature remains neutral with regard to jurisdictional claims in published maps and institutional affiliations.

Supplementary Information accompanies this paper at (https://doi.org/ 10.1038/s41419-018-0889-y).

Received: 23 June 2018 Revised: 17 July 2018 Accepted: 17 July 2018 Published online: 01 August 2018

\section{References}

1. Dalod, M., Chelbi, R., Malissen, B. \& Lawrence, T. Dendritic cell maturation: functional specialization through signaling specificity and transcriptional programming. EMBO J. 33, 1104-1116 (2014).

2. Banchereau, J. et al. Immunobiology of dendritic cells. Annu. Rev. Immunol. 18 767-811 (2000).

3. Cools, N., Ponsaerts, P., Van Tendeloo, V. F. \& Berneman, Z. N. Balancing between immunity and tolerance: an interplay between dendritic cells, regulatory T cells, and effector T cells. J. Leukoc. Biol. 82, 1365-1374 (2007).

4. Lee, E. G. et al. Tolerogenic dendritic cells show gene expression profiles that are different from those of immunogenic dendritic cells in DBA 1 mice. Autoimmunity 49, 90-101 (2016).

5. Nasr, N. et al. HIV-1 infection of human macrophages directly induces viperin which inhibits viral production. Blood 120, 778-788 (2012).
6. Helbig, K. J. et al. The antiviral protein viperin inhibits hepatitis $C$ virus replication via interaction with nonstructural protein 5A. Hepatology 54, 1506-1517 (2011).

7. Honarmand Ebrahimi, K. A unifying view of the broad-spectrum antiviral activity of RSAD2 (viperin) based on its radical-SAM chemistry. Metallomics 10, 539-552 (2018).

8. Severa, M., Coccia, E. M. \& Fitzqerald, K. A. Toll-like receptor-dependent and -independent viperin gene expression and counter-regulation by PRDIbinding factor-1/BLIMP1. J. Biol. Chem. 281, 26188-26195 (2006).

9. Mattijssen, S. \& Pruijn, G. J. Viperin, a key player in the antiviral response. Microbes Infect. 14, 419-426 (2012).

10. Lesage-Padilla, A. et al. Maternal metabolism affects endometrial expression of oxidative stress and FOXL2 genes in cattle. PLOS ONE 12, e0189942 (2017).

11. Zahoor, M. A. et al. HIV-1 Vpr induces interferon-stimulated genes in human monocyte-derived macrophages. PLoS ONE 9, e106418 (2014).

12. Teng, T. S. et al. Viperin restricts chikungunya virus replication and pathology. J. Clin. Invest. 122, 4447-4460 (2012).

13. Akira, S., Saitoh, T. \& Kawai, T. Nucleic acids recognition by innate immunity. Uirusu 62, 39-45 (2012)

14. Baranek, T., Zucchini, N. \& Dalod, M. Plasmacytoid dendritic cells and the control of herpes virus infections. Viruses 1, 383-419 (2009).

15. Robbins, S. H. et al. Novel insights into the relationships between dendritic cell subsets in human and mouse revealed by genome-wide expression profiling. Genome Biol. 9, R17 (2008).

16. Kapsenberg, M. L. Dendritic-cell control of pathogen-driven T-cell polarization. Nat. Rev. Immunol. 3, 984-993 (2003).

17. Honda, $\mathrm{K}$. et al. Selective contribution of IFN- $\alpha / \beta$ signaling to the maturation of dendritic cells induced by double-stranded RNA or viral infection. Proc. Nat Acad. Sci. USA 100, 10872-10877 (2003).

18. Alsharifi, M., Müllbacher, A. \& Regner, M. Interferon type I responses in primay and secondary infections. Immunol. Cell Biol. 86, 239-245 (2008).

19. Mattei, F., Bracci, L., Tough, D. F., Belardelli, F. \& Schiavoni, G. Type I IFN regulate DC turnover in vivo. Eur. J. Immunol. 39, 1807-1818 (2009).

20. Montoya, M. et al. Type I interferons produced by dendritic cells promote their phenotypic and functional activation. Blood 99, 3263-3271 (2002).

21. Sim, W. J., Malinarich, F., Fairhurst, A. M. \& Connolly, J. E. Generation of immature, mature and tolerogenic dendritic cells with differing metabolic phenotypes. J. Vis. Exp. 112, e54128 (2016).

22. Lazear, H. M. et al. IRF-3, IRF-5, and IRF-7 coordinately regulate the type I IFN response in myeloid dendritic cells downstream of MAVS signaling. PLoS. Pathog. 9, e1003118 (2013).

23. Klechevsky, E. Functional diversity of human dendritic cells. Adv. Exp. Med. Biol. 850, 43-54 (2015).

24. Longhi, M. P. et al. Dendritic cells require a systemic type I interferon response to mature and induce CD4+Th1 immunity with poly IC as adjuvant. J. Exp. Med. 206, 1589-1602 (2009).

25. Cunningham, C. R. et al. Type I and type II interferon coordinately regulate suppressive dendritic cell fate and function during viral persistence. PLoS. Pathog. 12, e1005356 (2016).

26. Robb, R. J. \& Hill, G. R. The interferon-dependent orchestration of innate and adaptive immunity after transplantation. Blood 119, 5351-5358 (2012).

27. Saitoh, T. et al. Antiviral protein Viperin promotes Toll-like receptor 7 - and Tolllike receptor 9-mediated type I interferon production in plasmacytoid dendritic cells. Immunity 34, 352-363 (2011).

28. Hata, M. et al. Expression of Th2-skewed pathology mediators in monocytederived type 2 of dendritic cells (DC2). Immunol. Lett. 126, 29-36 (2009).

29. Pham, P. V. et al. A simple in vitro method for evaluating dendritic cell-based vaccinations. Onco. Targets Ther. 7, 1455-1464 (2014).

30. Byun, S. H. et al. Rosiglitazone-mediated dendritic cells ameliorate collageninduced arthritis in mice. Biochem. Pharmacol. 115, 85-93 (2016). 